\title{
EXPRESIONES DEL CÁLCULO EN LA CULTURA MISKITU DE SANDY BAY SIRPI, REGIÓN AUTÓNOMA ATLÁNTICO SUR
}

Silguian Y. Gutiérrez M. ${ }^{[1]}$ Napoleón. Rojas R. ${ }^{[2]}$

\section{Resumen}

El propósito de esta investigación fue contribuir a la pertinencia de la enseñanza de las matemáticas desde las expresiones de cálculo en la cultura miskitu de la comunidad de Sandy Bay Sirpi. La metodología fue cualitativa etnográfica con una descripción amplia de las principales expresiones del cálculo de la cultura miskitu. Las técnicas y herramientas correspondieron a la observación, entrevista a profundidad, grupos focales y el uso de diario de campo para sistematizar los diferentes elementos de cotidianidad y cosmovisión miskitu de Sandy Bay Sirpi.

Los principales resultados, se han clasificado en seis actividades etnomatemáticas fundamentales y universales de grupos culturales, tales como contar, localizar, medir, diseñar, jugar y explicar. En el conteo, el pueblo miskitu tiene desde tiempos antiguos su propia numeración en idioma miskitu. De igual forma, realizan mediciones tanto de longitud como de masa y peso, utilizando, las partes del cuerpo (manos, pies y dedos), materiales como guacales y madera. En cuanto a las medidas del tiempo: El sol, así como el ciclo lunar y el calendario miskitu, son las principales estrategias de localización. Por otro lado, en la construcción de viviendas, cayucos y otros se utilizan diferentes figuras geométricas.

Palabras clave: Cálculos, conocimientos, cultura, etnomatemática, interculturalidad, matemática.

\section{Summary}

The purpose of this research was to contribute to the pertinence of teaching mathematics based on the expressions of calculus in the Miskitu culture of Sandy Bay Sirpi community. The methodology was qualitative ethnographic with a broad description of the main expressions of calculus in the Miskitu culture. The techniques and tools

[1] Máster en Educación Intercultural Multilingüe. Docente horario URACCAN Bluefields, silguiangutierrez@gmail.com

[2] Máster en Docencia Universitaria, tutor de la tesis. Coordinador del área de Humanidades de URACCAN Nueva Guinea, jnrr61@yahoo.com 
that were used corresponded to observation, profound interviews, focus groups and field notes, in order to systematize the different elements of everyday life and the cosmovision of the Miskitus of Sandy Bay Sirpi.

The main results have been classified into six fundamental and universal ethnomathematics activities of cultural groups, such as to count, locate, measure, design, play and explain. In counting, since ancient times, the Miskitu people have their own numbering in their language. Similarly, they perform measurements of length, mass and weight, using parts of the body (hands, feet, fingers), and materials such as wood crates. According to time measures: the sun, the lunar cycle and the Miskitu calendar are the main strategies of localization. On the other hand, for house constructions, canoes and others, different geometric shapes are used.

Keywords: Calculus, knowledge, culture, ethnomathematics, interculturality, mathematics.

\section{Introducción}

En Nicaragua la Educación Intercultural Bilingüe ha dado los primeros pasos, principalmente en las Escuelas Normales, universidades y algunas escuelas de Educación Primaria. Sin embargo, la matemática se sigue enseñando de forma aislada a la realidad cultural y lingüística, sin la inclusión de programas y planes educativos pertinentes a la realidad socio-cultural de cada territorio.

La presente investigación contribuye a la pertinencia de la enseñanza de las matemáticas desde las expresiones de cálculo en la cultura miskitu de la comunidad de Sandy Bay Sirpi. Se identifican las manifestaciones culturales relacionadas al cálculo matemático. Es decir, se describen las diferentes expresiones matemáticas desde la cultura en su propio contexto sociocultural, basadas en la etnografía de la comunidad de estudio. Sus resultados son un insumo fundamental para desarrollar la educación intercultural desde la etnomatemática del pueblo miskitu de Sandy Bay Sirpi. Por tal razón, se valora la inclusión de estos contenidos y medios de enseñanza en la educación formal, específicamente los contenidos y planes de clase de la Educación Primaria.

\section{Revisión de literatura}

\section{Educación Intercultural Bilingüe}

La Educación Intercultural Bilingüe es la educación centrada en la diferencia y pluralidad cultural más que una educación para los que son culturalmente diferentes. Los hombres y las mujeres de otras culturas son seres humanos, personas con las que construiremos una sociedad distinta y nueva (Sáez, 2005). Entonces, se puede 
afirmar que la educación intercultural es aquella práctica educativa que a partir de las relaciones asimétricas que se dan en la sociedad, establece un sistema de conocimientos y de valores, el que aspira construir relaciones equitativas entre los actores sociales y culturales.

La Educación Intercultural Bilingüe (ЕIB) es: "el proceso a través del cual los individuos al mismo tiempo que recuperan los conocimientos, saberes y tecnologías propios de su cultura, integran de manera crítica los conocimientos más importantes de la ciencia y la tecnología occidentales que les permitan construir formas de desarrollo sostenible y con identidad" (Diseño curricular Maya, 2010).

Villavicencio (2011), señala que la La Educación Intercultural Bilingüe (EIB) es el modelo educativo que se genera como repuesta al derecho de los pueblos originarios a una educación pertinente de calidad, que promueve la revaloración, el rescate y desarrollo de la cultura y lenguas originarias, y el reforzamiento de la identidad cultural en el marco del respeto, la reciprocidad, el diálogo, la interacción y mutuo aporte entre culturas.

La EIB para el caso particular de la costa Caribe nicaragüense, está sustentada en la constitución política, principalmente en su artículo 9o: "Las comunidades de la Costa Atlántica tienen derecho a la libre expresión y preservación de sus lenguas, arte y cultura (...)". El Estado creará programas especiales para el ejercicio de estos derechos. Por ende, uno de estos programas ha sido la creación del Subsistema Educativo Autonómico Regional (SEAR), incluido en la Ley General de Educación.

\section{Matemática, cálculo y etnomatemática}

El nacimiento de un sistema de numeración, la forma en que se opera y cómo se resuelven situaciones problemáticas, son particulares de cada lugar y de cada grupo de personas las desarrollan en un momento específico. Por tanto, la comprensión plena de este sistema de numeración parte del contexto en el cual ha nacido, se ha desarrollado o se mantiene vigente (Lara, 2006).

Se suele denominar a la etnomatemática como el conjunto de ideas y actividades matemáticas que se realizan en un contexto cultural. Por tanto es el arte o técnica de entender, explicar, aprender sobre, el manejo natural, social y político del medio ambiente, dependiendo de procesos como contar, medir, clasificar, ordenar e inferir, lo cual resulta de grupos culturales muy bien identificados (D'Ambrosio, 2007).

En matemática el cálculo consiste en un algoritmo (un conjunto de instrucciones pre- establecidas) que permite anticipar el resultado que procederá de ciertos datos que se conocen con anticipación. Todas las actividades matemáticas, conllevan "cálculo", de igual forma en la etnomatemática. Basado en esto, el investigador Alan Bishop 
(2002), ha destacado en indagaciones etnomatemáticas, que existen seis actividades etnomatemáticas fundamentales que son universales y que se encuentra presente en todos los grupos culturales. Estas corresponden a contar, localizar, medir, diseñar, jugar y explicar, todas ellas en algún momento también necesitan del proceso llamado cálculo.

\section{Materiales y métodos}

La investigación se desarrolló en la comunidad de Sandy Bay Sirpi, principal asentamiento de la población miskitu en el Atlántico sur de Nicaragua. De igual forma, fue cualitativa etnográfica, ya que se realizaron descripciones sobres los principales elementos culturales del pueblo miskitu de Sandy Bay Sirpi, para identificar las principales expresiones de cálculo. Para lo anterior se desarrollaron la observación, entrevista a profundidad, grupos focales como técnicas y herramientas de investigación. También se utilizó el diario de campo para sistematizar los diferentes elementos de la cultura y cosmovisión de este pueblo.

Los principales actores de este estudio fueron los ancianos de la comunidad, principalmente los organizados en el Consejo de Ancianos de la comunidad, conformado por hombres y mujeres mayores a 50 años de edad. Así mismo, los líderes comunitarios, las niñas y niños, en actividades cotidianas, las mujeres en las actividades hogareñas, los pescadores, artesanos, comerciantes, en sus labores propias, entre otros, todos aquellos grupos con los que se pudo interactuar durante las diferentes visitas a la comunidad.

Los principales criterios de inclusión para los actores claves del estudio, correspondieron a los siguientes:

- Que pertenezcan a la etnia miskitu.

- Que habite la comunidad de Sandy Bay Sirpi.

- Que posea conocimientos de los saberes tradicionales.

- Que sea líder de algún grupo observado: pescador, comerciante, político, consejo de ancianos, jóvenes, entre otros.

- Que tenga conocimiento del cálculo tradicional de la cultura miskitu.

El procesamiento y análisis se desarrolló mediante el diseño de una matriz según los descriptores del estudio en Word. 


\section{Resultados y discusión}

Los resultados de la investigación se han ordenado, según las seis actividades etnomatemáticas fundamentales y universales de grupos culturales. Entre estas, el contar, localizar, medir, diseñar, jugar y explicar.

\section{El conteo desde el pueblo Miskitu de Sandy Bay Sirpi}

El pueblo miskitu de Sandy Bay Sirpi cuenta con su propio sistema de numeración tradicional. Este le permite, cuantificar y medir los elementos importantes para su cultura. El conteo, lo realizan con las partes de su cuerpo, es decir, manos, pies, extremidades. La enumeración, es interesante analizarla desde la formación de las palabras que designan el número, porque ellas son la base del sistema numérico. A continuación, se detallan los números del 1 al 20:

$1=K u m i$

$2=w \hat{a} l$

3= yumhpa

4= walhwal

$5=$ Matsip

6= Matlalkahbi

7= Matlalkahbi pura kumi

$8=$ Matlalkahbi pura wâl

9= Matlalkahbi pura yumhpa

10= Matawalsip
11= Matawalsip pura kumi

12= Matawalsip pura wâl

13= Matawalsip pura yumhpa

14= Matawalsip pura walhwal

15= Matawalsip pura Matsip

16= Matawalsip pura matlalkahbi

17= Matawalsip pura matlalkahbi pura kumi

$18=$ Matawalsip pura matlalkahbi pura wâl

19= Matawalsip pura matlalkahbi pura yumhpa

20= Yawanaiska (yawan aiska)

Los anteriores, corresponde a kumi primer dedo, wâl segundo dedo, yumhpa tercero y así sucesivamente lo narran los ancianos. Posteriormente, al llegar a cinco es matsip, lo que representa la mano completa. El Diez es una suma de $5+5$, es decir dos manos y en la palabra diez se puede evidenciar esta suma matawalsip.

Posteriormente la suma continúa de cinco en cinco, simbolizando los dedos de los pies, hasta llegar a 20, por lo que a partir de allí, se denomina yawanaiska, que significa un entero. Posteriormente, las sumas se reanudan de cinco, hasta llegar a diez, aquí se forma el treinta con una combinación de un entero más 10, quedando de la siguiente forma: 
$30=20+10 \quad$ Treinta $(30)=$ Yawanaiska pura matawalsip;

$31=20+10+1, \ldots$ así $39=20+10+9$

Y luego $40=20+20 \quad$ Cuarenta $(40)=$ Yawanaiska wâl (dos enteros, o dos 20)

$50=20+20+10 \quad$ Cincuenta $(50)=$ Yawanaiska wâl pura matawalsip

Sesenta (6o) = Yawanaiska yumhpa

Setenta (70) = Yawanaiska yumhpa pura matawalsip

Los números se van formando matemáticamente según la gramática del miskitu, por lo que refieren cálculos matemáticos de sumas consecutivas, teniendo en cuenta los antecesores y sucesores. En este mismo conteo, el número 100 representa por ejemplo 5 personas, es decir cinco enteros, pero éste inicia con "handat; dusa kum", por así decirlo, con un nuevo conteo, nueva agrupación.

\section{El desarrollo de la medición y localización desde el pueblo miskitu de Sandy Bay Sirpi}

Las mediciones son el resultado de procesos de cálculo matemático, y estas se llevan a cabo en las construcciones, en la elaboración de ropas de vestir, en el comercio, entre otras actividades cotidianas. El pueblo miskitu, lo realiza de manera tradicional, con equivalencias correspondientes a unidades de medidas inglesas, tales como la yarda, los pies, pulgadas y varas para longitud, libras y onzas, respectivamente.

Para tales mediciones, utiliza el cuerpo, tales como por ejemplo, media brazada corresponde a una yarda, una brazada a dos yardas. La separación de los dedos, corresponde a una pulgada; las manos empuñadas y unidas por los dedos pulgares a un pie, doce pulgadas.

En el comercio y específicamente en la venta de madera, es utilizado el Pie cúbico $(12 \times 12 \times 1)$. Por lo que las tres mediciones en los cortes de madera obedecen a unidades de longitud del sistema inglés. Estos, lamentablemente no se ejercitan en la escuela; sin embargo, es una de las principales actividades económicas de la comunidad, por lo que sería importante su inclusión en el proceso educativo. Para ello, a continuación se presenta un ejemplo sobre la cubicación de madera para la construcción de un techo de una casa: 


\section{REVITALIZACIÓN LINGÜÍSTICA Y CULTURAL}

Cuadro No. 1. Mediciones del pueblo miskitu de Sandy Bay Sirpi

\begin{tabular}{|c|l|c|c|c|}
\hline $\begin{array}{c}\text { Nombres } \\
\text { comunes }\end{array}$ & \multicolumn{1}{|c|}{ Largo (pies) } & $\begin{array}{c}\text { Ancho } \\
\text { (pulgadas) }\end{array}$ & $\begin{array}{c}\text { Grosor } \\
\text { (pulgadas) }\end{array}$ & $\begin{array}{c}\text { Pies cúbicos } \\
\text { (Proceso de } \\
\text { cálculo) }\end{array}$ \\
\hline $2 \times 2$ & $\begin{array}{l}\text { Al gusto del cliente } \\
\text { (normalmente entre 12 y 16 } \\
\text { pies) }\end{array}$ & 2 pulg & 2 pulg & $\begin{array}{l}(12 \times 2 \times 2) / 12=4 \text { pies } \\
(16 \times 2 \times 2) / 12=5.3 \text { pies }\end{array}$ \\
\hline $4 \times 2$ & $\begin{array}{l}\text { lgual a la medida que dispon- } \\
\text { ga el cliente (Comunmente } \\
\text { entre 12 pies y 8 pies, en } \\
\text { coincidencia con el tamaño } \\
\text { de los zinc. }\end{array}$ & 4 pulg & 2 pulg & $\begin{array}{l}(12 \times 4 \times 2) / 12=8 \text { pies } \\
(8 \times 4 \times 2) / 12=5.3 \text { pies }\end{array}$ \\
\hline $4 \times 4$ & $\begin{array}{l}\text { Normalmente para hacer } \\
\text { cercos, entre 8y 10. }\end{array}$ & 4 pulg & 4 pulg & $\begin{array}{l}(8 \times 4 \times 4) / 12=5.3 \text { pies } \\
(10 \times 4 \times 4) / 12=13.3 \text { pies }\end{array}$ \\
\hline
\end{tabular}

En relación a las medidas de masa o peso, en su mayoría la comunidad utiliza las pesas tradicionales. Estas se elaboran con guacal y un trozo de madera, funcionando como balanza. Para ello, a continuación, se presenta una figura de la pesa:

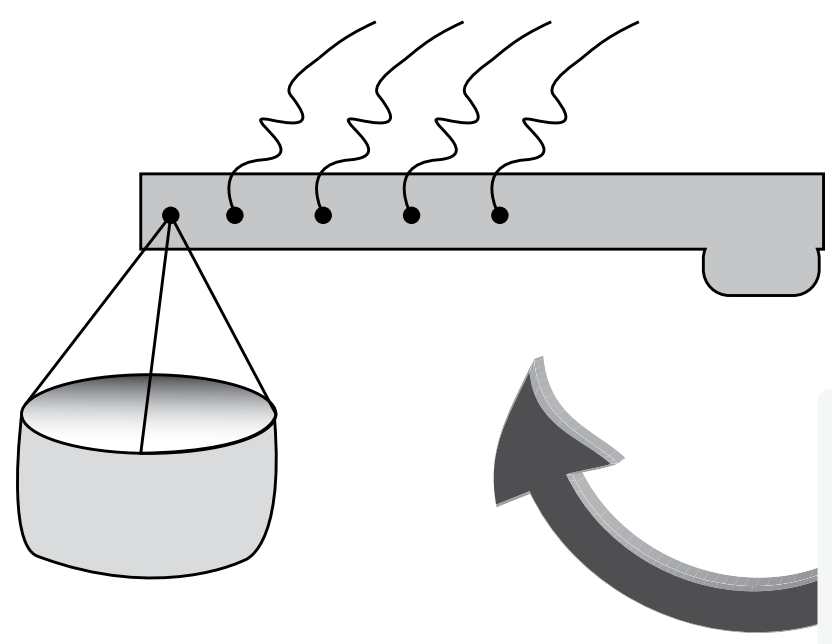

Cada separación entre una cuerda y otra debe tener una pulgada, también cada cuerda representa una libra cuando quedaba balanceada con el trozo de madera. 
Según los pescadores, esta pesa es utilizada para productos entre 5 y 10 libras. Sin embargo, en la actualidad, éstas se han venido cambiando por las balanzas mecánicas y electrónicas.

Por otro lado, en relación a las mediciones del tiempo, el pueblo miskitu de Sandy Bay Sirpi, cuenta con un calendario, el cual hace referencia a cada época del año. También se ha designado por la observación, cálculo y abundancia (presencia) de algunas especies de animales en los diferentes meses del año.

Enero: Siakwa kati, Siakwa es una tortuga de agua dulce asegura el informante, es la época de la caza de las tortugas.

Febrero: Kuswa Kati, tiempo de la tortuga jicotea o tortuga de tierra.

Marzo: Kakamuk Kati, tiempo de la iguana.

Abril: Waintka Kati, Pisma Kati, tiempo masculino.

Mayo: Wli Marin Kati, tiempo femenino; tiempo de la gran tortuga verde (marina); debido a las primeras lluvias de este mes, es propicio para los cultivos, por eso es el tiempo femenino (fertilidad).

Junio: Li Katii, mes del agua.

Julio: Pastara Kati, tiempo del viento fuerte.

Agosto: Sikla Kati, tiempo de los pájaros sigla.

Septiembre: Sikla Kati, tiempo del pájaro güis.

Octubre: Prari Kati, tiempo del huracán.

Noviembre: Yahbra Kati, tiempo del viento del Norte.

Diciembre: Krismas Kati, mes del Jesucristo.

También los meses del año lo podemos relacionar con el ciclo lunar, ya que en el idioma miskitu se designa por el término Kati a la luna y está presente en la formación de palabras que representan los nombres de los meses del año.

En relación al tiempo, además del calendario, los ancianos de la comunidad aún siguen las horas del día por la posición del sol. Es decir, la observación de las distintas posiciones que va teniendo el sol durante el día y la variación que tienen las sombras 
de los objetos expuestos a él, son las bases para ir midiendo las horas del día. También, los ancianos, mencionan que antes se utilizaban los nudos de las cuerdas para contar días de ausencia. Estas dos formas de medir el tiempo, en la actualidad, están siendo sustituidas por calendarios, relojes y otros accesorios de la sociedad moderna.

\section{Los juegos en los principios etnomatemáticos en Sandy Bay Sirpi}

La comunidad en sus diferentes actividades socioeconómicas, hacen uso de diversos elementos matemáticos. Entre estos, durante la elaboración de la red o atarraya para pescar camarones, en donde las mediciones del tamaño se hacen en base a la estatura de la persona que la usará. Además de esta medición, es importante mencionar que el tejido de la misma se desarrolla en forma de rombos simétricos unidos por nudos. Ambos elementos, de medición y diseño, deben ser retomados en la enseñanza de la matemática, ya que la pesca en la comunidad es una de las principales actividades económicas.

\section{Propuesta de inclusión de las expresiones de cálculo de la cultura miskitu a la Educación Intercultural Bilingüe y Básica}

Los conocimientos matemáticos de la cultura miskitu, citados y referidos en el presente trabajo de investigación, se pueden relacionar con los contenidos de los programas educativos del Ministerio de Educación (MINED). Estos corresponden a:

1. Cálculo mental.

2. Cálculo del tiempo.

3. Mediciones.

4. Cálculo proposicional (conjeturas).

5. Conteo.

6. Localizaciones, diseños de formas geométricas.

7. Elaboración de instrumentos de pesca.

Estos conocimientos son generales, pero cada situación puede ser abordada desde una visión etnomatemática en la escuela primaria. Por tanto, se recomiendan las siguientes actividades para su inclusión: 


\section{Propuesta $n^{\circ} 1$}

Contenido: Números del $1 \mathrm{al} 100$.

Competencia: Aplica la lógica del conteo oral en idioma miskitu y la relaciona con actividades de la vida real.

Indicador de logro: Construye la simbología de la numeración miskitu con distintos objetos, dibujos animales y materiales.

\section{Actividades sugeridas}

1) Contar con los estudiantes solamente en miskitu del 1 al 100, varios días, con actividades fuera del aula de clase, saliendo a recoger por orillas del mar conchas, o bien cantado. Así las niñas y niños aprenderán a contar del $1 \mathrm{al}$ 100 en miskitu y no se les olvidará.

2) Los estudiantes escriben cada quien en sus cuadernos en idioma miskitu los números del 1 al 100 (pueden usar una tabla).

3) Orientar a los estudiantes que representen, mediante los números en miskitus, cantidades correspondientes a los mismos.

Ejemplo:

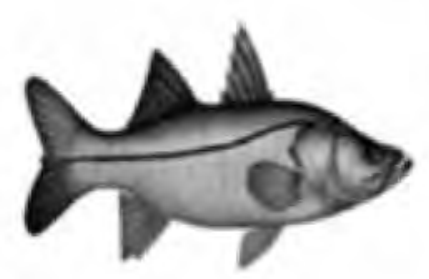

Kumi

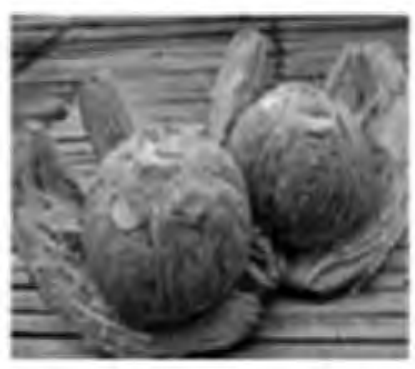

Wâl

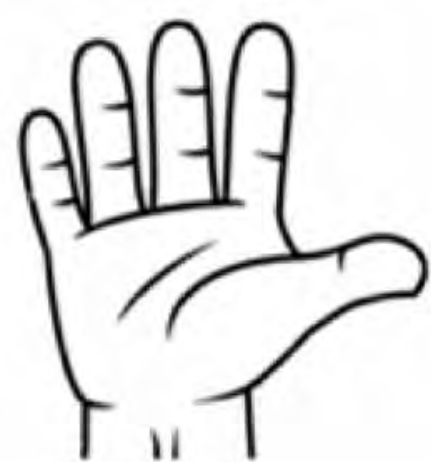

Matsip

4) Los docentes pueden reflexionar con los estudiantes alrededor de algunas situaciones con respecto al número y conteo en miskitu. Ejemplos de preguntas orientadoras: ¿Cuáles son los diferentes usos del número? ¿En qué situaciones cotidianas se utiliza el número? ¿Entre las personas de la comunidad se usan los números en miskitu? ¿Hasta qué número saben contar? ¿Les gusta contar en miskitu, porqué? 
Materiales sugeridos: Piedras, hojas, semillas, caracoles, huevos, frutas, palitos, hilo, verduras, lana, pajillas, nudos en cuerdas, juguetes, botones, gráficas, pegamento, recortes, periódicos.

Después del conteo se desarrollan las Adiciones de una y dos cifras, se propone abordarlas de la siguiente manera:

1) Los estudiantes analizan como se forman en idioma miskitu los números, algunas preguntas de orientación: ¿Qué sucede cuando se cuenta seis en idioma miskitu? ¿Cómo se forma el número siete? ¿Qué palabra se usa para designar el número diez? ¿Cómo se nombra el número quince?, entre otras.

2) Los docentes inducen a los estudiantes que la formación de los números en miskitus son adiciones.

3) Orientar a los estudiantes a completar el siguiente cuadro:

\begin{tabular}{|c|c|c|}
\hline Número & $\begin{array}{l}\text { Representación en } \\
\text { idioma miskitu }\end{array}$ & $\begin{array}{c}\text { Algoritmo de } \\
\text { formación (Adicción) }\end{array}$ \\
\hline o & Apu & o nada único termino \\
\hline 1 & Kumi & 1 único término \\
\hline 2 & Wâl & 2 único término \\
\hline 3 & Yumhpa & 3 único término \\
\hline 4 & Walhwal & 4 único término \\
\hline 5 & Matsip & 5 único término \\
\hline 6 & Matlakahbi & 6 único término \\
\hline 7 & Matlakahbi pura kumi & $6+1=7$ explique \\
\hline 8 & Matlakahbi pura wâl & $6+2=8$ explique \\
\hline 9 & Matlakahbi yumhpa & $6+3=9$ explique \\
\hline 10 & Matawalsip & $\begin{array}{l}2 \text { (manos), medio entero } \\
5+5=10\end{array}$ \\
\hline 11 & Matawalsip pura kumi & $10+1=11$, explique \\
\hline 12 & Matawalsip pura wâl & complete \\
\hline 13 & Matawalsip pura Yumhpa & complete \\
\hline 20 & Yawanaiska (yawan aiska) & $\begin{array}{l}\text { (Un entero) } 20 \text { único } \\
\text { término }\end{array}$ \\
\hline 24 & Yawanaiska pura walhwal & $20+4=24$, explique \\
\hline 26 & $\begin{array}{l}\text { Yawanaiska pura mat- } \\
\text { lalkahbi }\end{array}$ & complete \\
\hline 27 & $\begin{array}{l}\text { Yawanaiska pura mat- } \\
\text { lalkahbi pura kumi }\end{array}$ & complete \\
\hline
\end{tabular}




\begin{tabular}{|c|c|c|}
\hline Número & $\begin{array}{l}\text { Representación en } \\
\text { idioma miskitu }\end{array}$ & $\begin{array}{c}\text { Algoritmo de } \\
\text { formación (Adicción) }\end{array}$ \\
\hline 30 & $\begin{array}{l}\text { Yawanaiska pura ma- } \\
\text { tawalsip }\end{array}$ & $\begin{array}{l}\text { Un entero más la mitad } \\
\text { de otro. } 20+10=30\end{array}$ \\
\hline 40 & Yawanaiska wâl & Dos enteros $20+20=40$ \\
\hline 45 & $\begin{array}{l}\text { Yawanaiska wâl pura } \\
\text { matsip }\end{array}$ & $20+20+5=45$, explique \\
\hline 50 & $\begin{array}{l}\text { Yawanaiska wâl pura } \\
\text { matawalsip }\end{array}$ & $40+10=50$ \\
\hline
\end{tabular}

4) Realizar cálculos mentales propios de la cultura miskitu, es decir completando de 10 , en 10.

5) Hacer ejercicios en base al funcionamiento del cálculo mental como por ejemplo: $23+17=40,(7+3=10$ y $10+20=30$, para obtener 40$)$, explicando de forma oral y escrita el proceso en idioma miskitu.

Habilidades alcanzadas: Construye la simbología de numeración miskitu con distintos objetos y materiales.

\section{Conclusiones}

En el marco etnomatemático, la comunidad de Sandy Bay Sirpi conserva actividades matemáticas tradicionales, que le ayudan a comprender la naturaleza y resolver sus necesidades. Entre estos, los elementos identificados en la cultura miskitu son los siguientes: precisión, predicción, orientación, conjeturas, deducciones, mediciones, inducciones, idioma, entre otras.

Las seis acciones matemáticas universales se desarrollan en las actividades comunitarias, tales como la pesca, comercio, labores hogareñas y juegos. Estas deben de incluirse en los programas educativos del MINED y por ende en la escuela, para ello se ha propuesto el conteo en idioma miskitu con las enumeraciones occidentales; el cálculo mental con las sumas y restas de dos cifras; la enumeración en idioma miskitu con las sumas aritméticas y las sucesiones; las mediciones con equivalencias y conversiones matemáticas.

En la investigación de identificó que los conocimientos etnomatemáticos no están presentes en la escuela, por lo que su incorporación es necesaria debido a la influencia de otras culturas. 


\section{Lista de referencia}

Bishop, A.J. (2000). Enseñanza de las matemáticas: ¿Cómo enseñar a todos los alumnos?” en Matemática y Educación: Retos y cambios desde una perspectiva internacional $1^{\mathrm{a}}$ edición, Barcelona España, Editorial GRAÓ.

D’Ambrosio U. (2007) Matemática como ciencia de la sociedad. $1^{\text {a }}$ edición,

IPILC-URACCAN (2001). Sistema Educativo Autonómico Regional SEAR.

Lara C.M (2006). Aritmética maya: un aporte al currículo. Comité Latinoamericano de matemática educativa. México.

SEAR (2006). Ley No. 582. Ley General de Educación.

Sáez A. R. (2005).Tendencias actuales de la educación intercultural. Aula Intercultural. Disponible en http://www.aulaintercultural.org/article.php3?id_article=37

Villavicencio U.M. R (2011). Las Etnomatemáticas en la Educación Intercultural Bilingüe de Perú: Avances y cuestiones a responder. XIII Conferencia interamericana de Educación Matemática, Brasil. 